\title{
$\mathrm{BMJ}$
}

\section{Tricyclic antidepressants and headaches: systematic review and meta-analysis}

Jeffrey L Jackson, director, ${ }^{4}$ William Shimeall, programme director, ${ }^{2}$ Laura Sessums, director, ${ }^{1}$ Kent I DeZee, programme director, general medicine fellowship, ${ }^{3}$ Dorothy Becher, research associate, ${ }^{1}$ Margretta Diemer, staff physician, ${ }^{1}$ Elizabeth Berbano, staff physician, ${ }^{1}$ Patrick G O'Malley, director, general internal medicine ${ }^{3}$

${ }^{1}$ General Medicine Division, Walter Reed Army Medical Center,

Washington, DC, USA

Internal Medicine Residency, National Naval Medical Center, Bethesda, MD, USA

${ }^{3}$ Uniformed Services University, Bethesda

${ }^{4}$ General Medicine Division, Zablocki VA, 5000 W National Avenue, Milwaukee, WI, 53295, USA

Correspondence to: J L Jackson Jeffrey.jackson6@va.gov

Cite this as: $B M J$ 2010;341:C5222 doi:10.1136/bmi.c5222

\section{ABSTRACT}

Objective To evaluate the efficacy and relative adverse effects of tricyclic antidepressants in the treatment of migraine, tension-type, and mixed headaches.

Design Meta-analysis.

Data sources Medline, Embase, the Cochrane Trials Registry, and PsycLIT.

Studies reviewed Randomised trials of adults receiving tricyclics as only treatment for a minimum of four weeks. Data extraction Frequency of headaches (number of headache attacks for migraine and number of days with headache for tension-type headaches), intensity of headache, and headache index.

Results 37 studies met the inclusion criteria. Tricyclics significantly reduced the number of days with tension-type headache and number of headache attacks from migraine than placebo (average standardised mean difference $-1.29,95 \%$ confidence interval -2.18 to -0.39 and -0.70 , -0.93 to -0.48 ) but not compared with selective serotonin reuptake inhibitors $(-0.80,-2.63$ to 0.02 and $-0.20,-0.60$ to 0.19$)$. The effect of tricyclics increased with longer duration of treatment $(\beta=-0.11,95 \%$ confidence interval -0.63 to -0.15 ; P 0.0005$)$. Tricyclics were also more likely to reduce the intensity of headaches by at least $50 \%$ than either placebo (tension-type: relative risk 1.41, 95\% confidence interval 1.02 to 1.89 ; migraine: $1.80,1.24$ to $2.62)$ or selective serotonin reuptake inhibitors $(1.73,1.34$ to 2.22 and $1.72,1.15$ to 2.55 ). Tricyclics were more likely to cause adverse effects than placebo $(1.53,95 \%$ confidence interval 1.11 to 2.12 ) and selective serotonin reuptake inhibitors $(2.22,1.52$ to 3.32 ), including dry mouth ( $\mathrm{P}<0.0005$ for both), drowsiness ( $\mathrm{P}<0.0005$ for both), and weight gain (P<0.001 for both), but did not increase dropout rates (placebo: $1.22,0.83$ to 1.80 , selective serotonin reuptake inhibitors: $1.16,0.81$ to 2.97 ).

Conclusions Tricyclic antidepressants are effective in preventing migraine and tension-type headaches and are more effective than selective serotonin reuptake inhibitors, although with greater adverse effects. The effectiveness of tricyclics seems to increase over time.

\section{INTRODUCTION}

Headaches are common and cause distress and disability. The prevalence of migraine headaches ranges between $8.4 \%$ and $18 \%$ worldwide. ${ }^{12}$ Tension-type headaches are even more common, occurring in 16$30 \%$ of people worldwide, with $3 \%$ having headaches for more than 180 days a year. ${ }^{2}$ Migraine headaches alone cost the United States $\$ 1 \mathrm{bn}(£ 0.64 \mathrm{bn} ; € 0.75 \mathrm{bn})$ in medical costs and $\$ 13 \mathrm{bn}$ in lost productivity a year. ${ }^{3}$

Tricyclic antidepressants were first shown to be effective in preventing headaches in $1964^{4}$ and have become a standard modality in headache prevention. ${ }^{5}$ Based on current standards for preventive treatment in the United States, $43 \%$ of males and 34\% of females who are candidates for such treatment are not receiving it. ${ }^{6}$ This may result from insufficient understanding of the magnitude of beneficial effects, an overestimation of adverse effects, or the presumption that efficacy is only confined to migraine headaches. In a previous meta-analysis of antidepressants for headaches, we found that antidepressants were effective in preventing headaches, equally for tension-type headaches and migraine headaches. ${ }^{7}$ This meta-analysis was limited by the relatively small number of available studies at the time. To expand on our previous systematic review we assessed the efficacy and tolerability of tricyclics in reducing the headache burden among adults with migraine or tension-type headache. We also compared tricyclics with other treatment modalities to assess whether the efficacy of tricyclics varies by type of headache, dose, and duration of treatment.

\section{METHODS}

This report closely adheres to the PRISMA method for reporting on systematic reviews. We searched, without language restrictions, Medline (1966-March 2010) and Embase (1974-March 2010) using the search strategy (antidepressive agents, tricyclic OR antidepressive\$ OR tricyclic\$ OR amitriptyline OR amoxapine OR clomipramine OR desipramine OR dibenzepin OR dothiepin OR doxepin OR imipramine OR lofepramine OR nortriptyline OR opipramol OR protriptyline OR trimipramine) AND (headache or headache disorders or headache\$ OR migrain\$ OR tension\$ OR cephalgi\$ OR cephalalgi\$). We also searched CRISP and FEDRIP databases for unpublished 
literature. In addition we searched the Cochrane Pain, Palliative and Supportive Care Trials Register; the Cochrane Central Register of Controlled Trials; PsycLIT (1974-2002); and PsycINFO (1974-March 2010), and carried out a review of the bibliographies of all articles retrieved. The last search date was 25 March 2010. The search was supplemented by searches carried out by medical librarians at our institution as well as the Cochrane clinical research group.

We included published, randomised clinical trials that evaluated the efficacy of tricyclic antidepressants in reducing the frequency or severity of migraine or tension-type headaches. Treatment groups were required to receive a tricyclic regularly at any dosing schedule as a single intervention for at least four weeks. Tricyclics could not be combined with other drugs with possible prophylactic benefit or effect augmentation. Comparison groups could receive placebo or a specified alternative drug or non-drug treatment. Additional inclusion criteria required studies to include only adults $(>18$ years) with migraine or tension-type headache (frequent episodic or chronic) that could reasonably be defined on the basis of diagnostic criteria described in 1988 by the International Headache Society ${ }^{8}$ or earlier. ${ }^{910}$ We excluded secondary headaches, such as those related to drug overuse, concussion, or lumbar puncture. Because the classification of headache has changed over time, two authors independently reviewed each included article's definition of headache and, where possible, classified it according to the most recent criteria of the International Headache Society. ${ }^{8}$

\section{Study selection and data abstraction}

We selected articles for inclusion in two stages. In the first stage two researchers (PGO'M, KJD) independently reviewed titles and abstracts to select full text articles for retrieval. A priori we decided to include all articles recommended by either reviewer for full text retrieval. Agreement was only modest during this stage $(\kappa=0.66)$. During the second stage two other researchers (LS, MD) independently applied inclusion and exclusion criteria using standardised forms. Agreement was good $(\kappa=0.86)$; during this stage, disagreements were resolved by consensus.

Two authors (WS, JLJ) independently abstracted data using standardised forms. Data abstracted included information about the study (setting, country, language, inclusion and exclusion criteria), information about the intervention (design, characteristics of treatment, such as dose and duration), characteristics of the participants (age, sex, ethnicity, assessment of comorbid psychiatric disease), and treatment related patient reported outcomes for headache (frequency, intensity or severity, duration, global improvement or relief, analgesics used, adverse events). For continuous outcomes we abstracted the number of participants, the mean, and the variance. We calculated missing variances from reported $\mathrm{P}$ values. ${ }^{11}$ For dichotomous outcomes we abstracted data into $2 \times 2$ tables, requiring at least $50 \%$ clinical improvement in headache. Studies reporting less than 50\% improvement were not abstracted. We assessed article quality independently and in duplicate (DB, JLJ) using the risk of bias assessment tool developed by the Cochrane collaboration, ${ }^{12}$ with good inter-rater agreement (Cochrane: $\kappa=0.83$ ). Disagreements were resolved by consensus.

\section{Statistical analysis}

Because reported measures for headache varied, a priori we preferentially abstracted and pooled data on headache measures in this order: frequency of headache, intensity of headache, and headache index, per the recommendations of the reviewers of our Cochrane protocol. For frequency we used the number of headache attacks for migraines and number of days with headache for tension-type headaches. Intensity was abstracted in the scale used by each study; some studies used visual analogue scales, others used other continuous measures of intensity. Many studies used a "headache index" to sum aspects of the burden of headache, usually derived by multiplying the frequency of the headache by intensity. In addition we pooled analgesic use. Because of differences in measurement of headache burden between studies, we pooled standardised differences between studies, a measure also known as an effect size. By convention, effect sizes greater than 0.8 are considered as large, those between 0.6 and 0.8 as moderate, and those between 0.2 and 0.6 as small. ${ }^{13}$

For studies with more than one arm, we pooled the data for all arms into one group; if this was not possible we reduced the sample size for each arm by $50 \%$ to prevent overweighting studies. We pooled data using the DerSimonian and Laird random effects model. ${ }^{14}$ Regardless of headache type, we pooled all studies with stratified analysis to provide estimates for each subgroup, and used metaregression to test for differences between these groups. Heterogeneity was assessed visually using Galbraith plots ${ }^{15}$ and the $\mathrm{I}^{2}$ statistic. ${ }^{16}$ For continuous data (such as headache frequency and index) we used standardised mean differences (absolute differences divided by the standard deviation). We calculated the percentage maximum dose by dividing the maximum dose in each study by the maximum dose allowed for the study drugs. Quality assessment was done using a component approach. In addition we tested the effect of study sponsorship, use of intention to treat analysis, and whether adherence was assessed. Because of the large number of analyses, we used $\mathrm{P}=0.01$ as our threshold for significance. We assessed for small study effects (publication bias) using the methods of Peters ${ }^{17}$ for dichotomous outcomes and Eggers ${ }^{18}$ for continuous ones. We estimated the number of unpublished trials using Gleser's method $^{19}$ and assessed for the potential effects of bias using both the file drawer test ${ }^{20}$ and the trim and fill method. ${ }^{21}$ For the file drawer test we tested how many studies would be required to make our results clinically insignificant - that is, an effect size less than 0.30 . We explored the potential source of 
heterogeneity using stratified analysis and meta regression. $^{22}$ All analyses were done using Stata (v 11.2).

\section{RESULTS}

Overall, 1471 potential articles were identified. On the initial screen 443 full text articles were retrieved and assessed for eligibility (fig 1). Thirty seven studies met the inclusion criteria ${ }^{423-58} ; 13$ focused on migraine headaches, ${ }^{23242931323436404143505158} 17$ on tension-type headaches, ${ }^{4252628333537-39424445475355-57}$ six on chronic mixed headaches (components of migraine and tension-type headaches), ${ }^{273046484954}$ and one on psychogenic headaches. ${ }^{52}$ Six studies used the 1988 criteria of the International Headache Society, 11 used the 1962 ad hoc committee criteria, and the remainder used the most recent criteria. All trials of migraine headaches would meet the most recent criteria. Among trials of tension-type headache, one met the criteria for infrequent episodic headaches, six for frequent episodic headaches, and 15 for chronic headache according to the most recent criteria. The six trials with mixed headaches were analysed as migraine headaches as they better met the criteria for migraine headache than for tension-type headache.

Twenty nine of the 37 studies used parallel group designs and eight crossover designs. None of the crossover studies provided sufficient data from which to calculate paired within patient results. ${ }^{4529364348515859}$ All eight were, however, included as one trial, ${ }^{25}$ reported no carry-over effect, and the remainder had either more than four weeks of washout ${ }^{4358}$ or sufficiently long treatment durations to minimise carry-over effects. ${ }^{364348}$

A total of 3176 participants were enrolled across the 37 studies (table 1). Studies lasted an average of 10 weeks (range $4-26$ weeks). A mean 70 participants

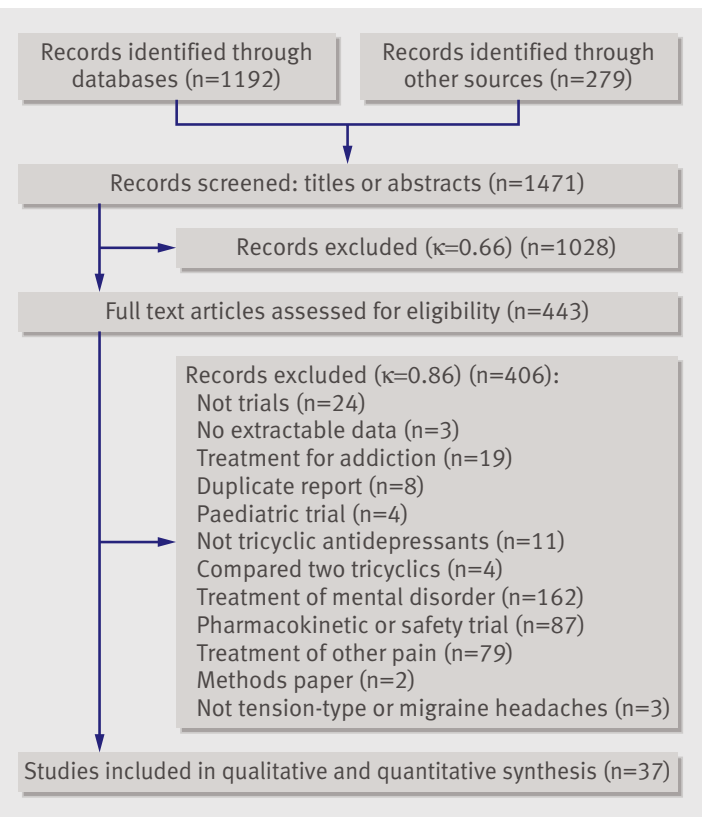

Fig 1| Flow of articles through study (range 10-554) took part in the studies. Seventy three per cent of participants were women (range 28-98\%); all but one study ${ }^{52}$ had more women than men. The mean age of participants was 39.6 years. Ten studies were carried out in the United States; five in Italy; four in the United Kingdom; three each in Denmark, Germany, and Turkey; two in Russia; and one each in Australia, Canada, Egypt, Greece, Hungary, Norway, and Spain. All but four studies were in English. ${ }^{23304555}$ Nine trials were sponsored by industry, and in 16 the source of funding was not stated.

Among the 37 studies, 20 compared a tricyclic with placebo. Some studies had more than one comparison group. A few studies compared tricyclics with other modalities, including eight with selective serotonin reuptake inhibitors, three with $\beta$ blockers, three with heterocyclics, and one each with buspirone, ${ }^{47}$ dihydroergotamine,${ }^{27}$ flunarizine,${ }^{30}$ and ritanserin. ${ }^{49}$ One study compared amitriptyline with cognitive behavioural therapy ${ }^{37}$ and one with stress management, ${ }^{38}$ one compared biofeedback with amitriptyline and propranolol, ${ }^{46}$ three compared amitriptyline with spinal manipulation, ${ }^{265056}$ and one compared amitriptyline and fluvoxamine with transcranial brain electrostimulation. ${ }^{55}$

Measures of headache included frequency of headache (19 studies), headache intensity ( $\mathrm{n}=5)$, and headache index $(n=13)$. All the studies using headache index used frequency of headache as one component in the index calculation.

Most studies ( $\mathrm{n}=29)$ titrated the study drugs. The maximum allowable daily dose for amitriptyline (30 studies) ranged from $10 \mathrm{mg}^{30}$ to $150 \mathrm{mg},{ }^{3358}$ whereas the four studies of clomipramine ranged from $30 \mathrm{mg}^{43}$ to $150 \mathrm{mg} .{ }^{42}$ Other maximum daily doses of tricyclics were opipramol $150 \mathrm{mg},{ }^{40}$ doxepin $100 \mathrm{mg},{ }^{48}$ amitriptylinoxide $90 \mathrm{mg},{ }^{483}$ and desipramine $150 \mathrm{mg} .{ }^{57}$ Periods of dose titration ranged from none $e^{2427304549}$ to four weeks. $^{333743535758}$ The mean pooled doses were amitriptyline $80.4 \mathrm{mg}$ (95\% confidence interval 70.1 to 90.7, range 25-150), amitriptylinoxide $90 \mathrm{mg}$ (one study), clomipramine $116 \mathrm{mg}$ (104 to 128, range 30150), doxepin $50 \mathrm{mg}(\mathrm{n}=1)$, and opipramol $75 \mathrm{mg}$ $(\mathrm{n}=1)$.

Seven studies excluded patients with depression. ${ }^{25282935475356}$ Eleven studies reported the number of patients at trial entry meeting criteria for depression, ranging from $21 \%$ to $98 \%$. The remaining studies did not measure or report on depression.

Dropout rates ranged from 0 to $52 \%$. Four studies had less than $10 \%$ losses to follow-up, ${ }^{28495354}$ eight between $10 \%$ and 20\%, ${ }^{252637-39414755}$ nine between $20 \%$ and 30\%, ${ }^{232427303642444650}$ eight between $30 \%$ and $40 \%,{ }^{2931-354857}$ and five more than 40\%. ${ }^{4043515658}$ Losses to follow-up could not be determined in three studies. ${ }^{484552}$ Intention to treat analyses were done in only seven trials. ${ }^{26334950535456}$

\section{Quality ratings}

Although quality varied among the studies, most had important limitations with design or reporting (table 2). 


\section{Table 1/Characteristics of included studies}

\begin{tabular}{|c|c|c|c|c|c|c|c|}
\hline Study (country) & Design, intervention (maximum dose) & $\begin{array}{l}\text { Duration of } \\
\text { treatment } \\
\text { (weeks) }\end{array}$ & $\begin{array}{l}\text { Headache } \\
\text { type }\end{array}$ & $\begin{array}{l}\text { No of } \\
\text { participants } \\
\text { (\% women) }\end{array}$ & Age (range) & $\begin{array}{l}\text { Drop outs } \\
\text { (\%) }\end{array}$ & Depression \\
\hline Amelin $2000^{23}$ (Russia) & $\begin{array}{l}\text { Parallel } 3 \text { arm comparing amitriptyline }(25 \mathrm{mg}) \text {, fluoxetine }(20 \mathrm{mg}) \text {, and } \\
\text { maprotiline }(25 \mathrm{mg})\end{array}$ & 12 & Migraine & $46(95)$ & $36(21-57)$ & 23 & $\begin{array}{l}\text { Hamilton depression } \\
\text { rating scale }\end{array}$ \\
\hline Bank $1994^{24}$ (Hungary) & Parallel 2 arm comparing amitriptyline ( $25 \mathrm{mg}$ ) with fluvoxamine $(50 \mathrm{mg})$ & 12 & Migraine & $49(80)$ & $34(20-62)$ & 23 & NS \\
\hline Bendtsen $1996^{25}$ (Denmark) & $\begin{array}{l}\text { Crossover } 3 \text { arm comparing amitriptyline }(75 \mathrm{mg}) \text {, citalopram }(20 \mathrm{mg}) \text {, and } \\
\text { placebo }\end{array}$ & 8 & Tension & $34(65)$ & $40(18-60)$ & 15 & Excluded \\
\hline Boline $1995^{26}$ (USA) & Parallel 2 arm comparing amitriptyline $(30 \mathrm{mg})$ with spinal manipulation & 6 & Tension & $126(61)$ & $42(18-69)$ & 16 & NS \\
\hline Bonuso $1983^{27}$ (Italy) & Parallel 2 arm comparing amitriptyline $(75 \mathrm{mg})$ with dihydroergotamine $(10 \mathrm{mg})$ & 8 & Mixed* & $30(68)$ & NS (18-55) & 27 & NS \\
\hline Boz $2003^{28}$ (Turkey) & Parallel 2 arm comparing amitriptyline $(25 \mathrm{mg})$ with sertraline $(50 \mathrm{mg})$ & 12 & Tension & $84(89)$ & $40.4(19-64)$ & 7 & Excluded \\
\hline Bulut $2004^{29}$ (Turkey) & Crossover 2 arm comparing venlafaxine $(150 \mathrm{mg})$ with amitriptyline $(75 \mathrm{mg})$ & 24 & Migraine & $52(85)$ & $31.9(16-50)$ & 32 & Excluded \\
\hline Canepari $1985^{30}$ (Italy) & Parallel 3 arm comparing amitriptyline $(10 \mathrm{mg})$, flunarizine $(10 \mathrm{mg})$, and placebo & 12 & Mixed* & $42(98)$ & $36(15-65)$ & 28 & NS \\
\hline Couch $1974^{31}$ (USA) & Parallel 2 arm comparing amitriptyline $(100 \mathrm{mg})$ with placebo & 4 & Migraine & $73(85)$ & NS (NS) & 36 & $\begin{array}{l}\text { Hamilton depression } \\
\text { rating scale, Zung } \\
\text { depression scale }\end{array}$ \\
\hline Couch $1976^{32}$ (USA) & Parallel 2 arm comparing amitriptyline $(100 \mathrm{mg})$ with placebo & 8 & Migraine & $100(84)$ & NS (NS) & 38 & $\begin{array}{l}\text { Hamilton depression } \\
\text { rating scale, Zung } \\
\text { depression scale }\end{array}$ \\
\hline Diamond $1971^{33}$ (USA) & $\begin{array}{l}\text { Parallel } 3 \text { arm comparing amitriptyline }(60 \mathrm{mg}) \text {, amitriptyline }(150 \mathrm{mg}) \text {, and } \\
\text { placebo }\end{array}$ & 4 & Tension & $85(75)$ & $46(18-68)$ & 32 & $\begin{array}{l}\text { Measured } \\
\text { (unspecified) }\end{array}$ \\
\hline Dodick $2009^{34}$ (USA) & Parallel 2 arm comparing topiramate $(100 \mathrm{mg})$ with amitriptyline $(100 \mathrm{mg})$ & 26 & Migraine & $331(85)$ & $38.8(18-70)$ & 40 & NS \\
\hline Gobel $1994^{35}$ (Germany) & Parallel 2 arm comparing amitryptiline $(75 \mathrm{mg})$ with placebo & 6 & Tension & $53(70)$ & $43(\mathrm{NS})$ & 32 & Excluded \\
\hline Gomersall $1973^{36}(\mathrm{UK})$ & Crossover 2 arm comparing amitryptiline $(60 \mathrm{mg})$ with placebo & 26 & Migraine & $20(75)$ & $42(21-70)$ & 23 & $\begin{array}{l}\text { Patients were asked if } \\
\text { they were depressed }\end{array}$ \\
\hline Holroyd $1991^{37}$ (USA) & Parallel 2 arm comparing amitriptyline $(75 \mathrm{mg})$ with cognitive behavioural therapy & 12 & Tension & $41(80)$ & $32.3(19-55)$ & 12 & $\begin{array}{l}\text { Beck depression } \\
\text { inventory }\end{array}$ \\
\hline Holroyd $2001^{38}$ (USA) & $\begin{array}{l}\text { Parallel } 4 \text { arm comparing amitriptyline }(100 \mathrm{mg}) \text { or nortriptyline }(100 \mathrm{mg}) \text {, stress } \\
\text { management therapy, amitriptyline or nortriptyline plus stress management } \\
\text { therapy, and placebo }\end{array}$ & 24 & Tension & $203(76)$ & 37 (NS) & 12 & NS \\
\hline Indaco $1988^{39}$ (Italy) & Parallel 2 arm comparing amitriptyline $(50 \mathrm{mg})$ with placebo & 12 & Tension & $31(52)$ & $60.4(41-70)$ & 14 & Zung depression scale \\
\hline Jacobs $1972^{40}(\mathrm{UK})$ & Parallel 2 arm comparing opipramol $(150 \mathrm{mg})$ with placebo & 12 & Migraine & $27(78)$ & $42(23-67)$ & 43 & NS \\
\hline Keskinbora $^{41} 2008$ (Turkey) & $\begin{array}{l}\text { Parallel } 3 \text { arm comparing amitriptyline }(150 \mathrm{mg}) \text {, topiramate }(200 \mathrm{mg}) \text {, and } \\
\text { combination of these two drugs }\end{array}$ & 12 & Migraine & $63(67)$ & 37 (NS) & 16 & $\begin{array}{l}\text { Beck depression } \\
\text { inventory }\end{array}$ \\
\hline Lance $1964^{4}$ (Australia) & Crossover 2 arm comparing amitriptyline $(75 \mathrm{mg})$ with placebo & 4 & Tension & $27(63)$ & NS (NS) & NS & NS \\
\hline Langemark $1990^{42}$ (Denmark) & Parallel 3 arm comparing clomipramine ( $150 \mathrm{mg})$, mianserin $(60 \mathrm{mg})$, and placebo & 6 & Tension & 82 (NS) & $41(18-69)$ & 28 & NS \\
\hline Langohr $1985^{43}$ (Germany) & $\begin{array}{l}\text { Crossover } 5 \text { arm comparing all crossover combinations of clomipramine (100 mg), } \\
\text { metoprolol (100 mg), and placebo }\end{array}$ & 4 & Migraine & $36(67)$ & $44(24-60)$ & 43 & NS \\
\hline Loldrup $1989^{44}$ (Denmark) & Parallel 3 arm comparing clomipramine $(150 \mathrm{mg})$, mianserin $(60 \mathrm{mg})$, and placebo & 6 & Tension & $114(64)$ & $41(17-69)$ & 20 & $\begin{array}{l}\text { Measured } \\
\text { (unspecified) }\end{array}$ \\
\hline Martin-Araguz $2003^{45}$ (Spain) & Parallel 2 arm comparing amitriptyline $(25 \mathrm{mg})$ with mirtazapine ( $30 \mathrm{mg})$ & 24 & Tension & $60(70)$ & 38 (NS) & NS & $\begin{array}{l}\text { Hamilton depression } \\
\text { rating scale }\end{array}$ \\
\hline Mathew $1981^{46}$ (USA) & $\begin{array}{l}\text { Parallel } 8 \text { arm comparing multi-intervention: amitriptyline }(75 \mathrm{mg}) \text {, propranolol } \\
(160 \mathrm{mg}) \text {, biofeedback, placebo, amitriptyline+propranolol, amitriptyline } \\
\text { +biofeedback, propranolol+biofeedback, and amitriptyline+propranolol } \\
\text { +biofeedback }\end{array}$ & 24 & $\begin{array}{l}\text { Migraine and } \\
\text { mixed }\end{array}$ & $554(95)$ & $38(19-57)$ & 22 & Zung depression scale \\
\hline Mitsikostas $1997^{47}$ (Greece) & Parallel 2 arm comparing amitriptyline ( $50 \mathrm{mg}$ ) with buspirone ( $30 \mathrm{mg}$ ) & 12 & Tension & $49(62)$ & 43 (NS) & 16 & $\begin{array}{l}\text { Hamilton depression } \\
\text { rating scale }\end{array}$ \\
\hline Morland $1979^{48}$ (Norway) & Crossover 2 arm comparing doxepin ( $100 \mathrm{mg}$ ) with placebo & 9 & $\begin{array}{l}\text { Mixed and } \\
\text { tension }\end{array}$ & $14(86)$ & NS (20-50) & 39 & NS \\
\hline Nappi $1990^{49}$ (Italy) & Parallel 2 arm comparing amitriptyline $(50 \mathrm{mg})$ with ritanserin $(10 \mathrm{mg})$ & 12 & $\begin{array}{l}\text { Migraine and } \\
\text { tension }\end{array}$ & $38(79)$ & $38(20-50)$ & 0 & $\begin{array}{l}\text { Hamilton depression } \\
\text { rating scale }\end{array}$ \\
\hline Nelson $1998^{50}$ (USA) & $\begin{array}{l}\text { Parallel } 3 \text { arm comparing amitriptyline }(100 \mathrm{mg}) \text {, spinal manipulation, and } \\
\text { amitriptyline+spinal manipulation }\end{array}$ & 8 & Migraine & $218(79)$ & 38 (NS) & 23 & NS \\
\hline Noone $1980^{51}(\mathrm{UK})$ & Crossover 2 arm comparing clomipramine $(30 \mathrm{mg}$ ) with placebo & 8 & Migraine & $10(70)$ & NS (18-65) & 52 & $\begin{array}{l}\text { Beck depression } \\
\text { inventory }\end{array}$ \\
\hline Okasha $1973^{52}$ (Egypt) & $\begin{array}{l}\text { Parallel } 4 \text { arm comparing amitriptyline }(30 \mathrm{mg}) \text {, doxepin }(30 \mathrm{mg}) \text {, diazepam } \\
(6 \mathrm{mg}) \text {, and placebo }\end{array}$ & 8 & Psychogenic & $80(28)$ & NS (16-60) & NS & $\begin{array}{l}\text { Hamilton depression } \\
\text { rating scale }\end{array}$ \\
\hline Pfaffenrath $1994^{53}$ (Germany) & $\begin{array}{l}\text { Parallel } 3 \text { arm comparing amitriptyline }(75 \mathrm{mg}) \text {, amitriptylinoxide }(90 \mathrm{mg}) \text {, and } \\
\text { placebo }\end{array}$ & 12 & Tension & $197(56)$ & $38(18-68)$ & 4.1 & Excluded \\
\hline Rampello $2004^{54}$ (Italy) & Parallel 2 arm comparing amitriptyline $(50 \mathrm{mg})$ with citalopram $(20 \mathrm{mg})$ & 16 & Mixed* & $88(63)$ & $39(22-64)$ & 0 & $\begin{array}{l}\text { Hamilton depression } \\
\text { rating scale }\end{array}$ \\
\hline Tarosova $2008^{55}$ (Russia) & $\begin{array}{l}\text { Parallel } 3 \text { arm comparing amitriptyline }(100 \mathrm{mg}) \text {, fluvoxamine }(100 \mathrm{mg}) \text {, and } \\
\text { transcranial brain electrostimulation }\end{array}$ & 4 & Tension & $44(90)$ & $44(34-60)$ & 18 & $\begin{array}{l}\text { Beck depression } \\
\text { inventory }\end{array}$ \\
\hline Vernon $2009^{56}$ (Canada) & $\begin{array}{l}\text { Parallel } 4 \text { arm comparing amitriptyline }(25 \mathrm{mg}) \text {, spinal manipulation, sham spinal } \\
\text { manipulation, and amitriptyline+spinal manipulation }\end{array}$ & 14 & Tension & $20(80)$ & $34.1(18-48)$ & 50 & Excluded \\
\hline Walker $1998^{57}(\mathrm{UK})$ & Parallel 2 arm comparing desipramine $(150 \mathrm{mg})$ with fluoxetine $(40 \mathrm{mg})$ & 12 & Tension & $25(81)$ & $35(19-59)$ & 32 & $\begin{array}{l}\text { Hamilton depression } \\
\text { rating scale }\end{array}$ \\
\hline Ziegler $1987^{58}$ (USA) & $\begin{array}{l}\text { Crossover } 3 \text { arm comparing amitriptyline }(150 \mathrm{mg}) \text {, propranolol }(240 \mathrm{mg}) \text {, and } \\
\text { placebo }\end{array}$ & 4 & Migraine & $30(73)$ & $38(22-57)$ & 44 & $\begin{array}{l}\text { Hamilton depression } \\
\text { rating scale, Zung } \\
\text { depression scale }\end{array}$ \\
\hline
\end{tabular}

*Analysed as migraine headache. 
Few studies reported the method of randomisation or allocation concealment. Although trials were often reported as double blind, blinding success was reported in only one trial ${ }^{57}$ and was often questionable, as treatment groups differed significantly in clinically recognisable adverse effects.

\section{Headache outcomes}

Tricyclics versus placebo

At baseline, participants with migraine averaged 4.7 headaches per month $(95 \%$ confidence interval 4.3 to 5.1) and those with tension-type headaches 16.9 (15.8 to 18.0), with no difference between tricyclic and placebo groups $(\mathrm{P}=0.46)$. Tricyclics were more effective than placebo in reducing the burden from both tension-type headaches (average standardised mean difference $-0.99,95 \%$ confidence interval -1.66 to $-0.32 ; 11$ arms, $\left.\mathrm{I}^{2}=93.8 \%\right)$ and migraine headaches $\left(-1.00,-1.52\right.$ to $-0.48 ; 10$ arms, $\mathrm{I}^{2}=89.4 \%$, fig 2$)$. Tricyclics were equally efficacious between the two headache types. The studies combined yielded an overall average standardised mean difference for tricyclics of $-0.96(95 \%$ confidence interval -1.39 to -0.53 , $\left.\mathrm{I}^{2}=90.1 \%\right)$. Therefore, regardless of the scale used to measure headaches, patients treated with a tricyclic experienced nearly 1 standard deviation of improvement, a clinically large effect. Efficacy did not differ among the different categories of tension-type

\begin{tabular}{|c|c|c|c|c|c|c|c|c|c|}
\hline Study & $\begin{array}{l}\text { Drop outs } \\
\text { (\%) }\end{array}$ & $\begin{array}{c}\text { Adherence } \\
\text { measured }\end{array}$ & $\begin{array}{l}\text { Adequate } \\
\text { sequence } \\
\text { generation }\end{array}$ & $\begin{array}{l}\text { Allocation } \\
\text { concealment }\end{array}$ & Blinding & $\begin{array}{c}\text { Incomplete } \\
\text { data } \\
\text { addressed }\end{array}$ & $\begin{array}{l}\text { Free of } \\
\text { selective } \\
\text { reporting }\end{array}$ & $\begin{array}{c}\text { Free of } \\
\text { other bias }\end{array}$ & $\begin{array}{l}\text { Industry } \\
\text { sponsored }\end{array}$ \\
\hline Amelin $^{23}$ & 23 & No & No & Unclear & No & No & Unclear & Yes & No \\
\hline Bank $^{24}$ & 23 & No & Unclear & Unclear & Unclear & No & Unclear & Yes & NS \\
\hline Bendtsen $^{25}$ & 15 & Yes & Unclear & Unclear & Yes & No & Unclear & Yes & No \\
\hline Boline $^{26}$ & 16 & Yes & Yes & Yes & No & No & Unclear & No & No \\
\hline Bonuso $^{27}$ & 27 & No & Unclear & Unclear & No & No & No & Yes & NS \\
\hline $\mathrm{Boz}^{28}$ & 7 & Yes & Unclear & Unclear & No & Yes & Unclear & Yes & No \\
\hline Bulut $^{29}$ & 32 & No & Unclear & Unclear & Yes & No & Unclear & Yes & Yes \\
\hline Canepari $^{30}$ & 28 & Yes & No & Unclear & Yes & No & Unclear & Yes & NS \\
\hline Couch $^{31}$ & 36 & No & Unclear & Unclear & Yes & No & Unclear & Yes & NS \\
\hline Diamond $^{33}$ & 6 & No & Unclear & Unclear & Yes & Yes & Unclear & Yes & NS \\
\hline Dodick $^{34}$ & 43 & No & Yes & Yes & Yes & Yes & Yes & Yes & Yes \\
\hline Gobel $^{35}$ & 32 & No & Unclear & Unclear & Yes & No & Unclear & Yes & NS \\
\hline Gomersall $^{36}$ & 23 & No & Unclear & Unclear & Unclear & No & No & Yes & Yes \\
\hline $\begin{array}{l}\text { Holroyd } \\
1991^{37}\end{array}$ & 12 & No & Unclear & Unclear & No & No & Unclear & Yes & NS \\
\hline $\begin{array}{l}\text { Holroyd } \\
2001^{38}\end{array}$ & 12 & Yes & Yes & Unclear & No & Yes & Yes & Yes & No \\
\hline Indaco 39 & 14 & Yes & Unclear & Unclear & Yes & No & Unclear & Yes & NS \\
\hline $\operatorname{Jacobs}^{40}$ & 43 & No & Unclear & Yes & Yes & No & No & Yes & Yes \\
\hline Keskinbora $^{41}$ & 16 & Yes & Unclear & Yes & Yes & No & Yes & Yes & NS \\
\hline Lance $^{4}$ & 0 & No & No & Unclear & Yes & Yes & Unclear & Yes & Yes \\
\hline Langemark $^{42}$ & 28 & No & Unclear & Unclear & Yes & No & No & Yes & Yes \\
\hline Langohr $^{43}$ & 43 & No & Unclear & Unclear & Unclear & No & No & Yes & Yes \\
\hline Loldrup $^{44}$ & 20 & Yes & Unclear & Unclear & No & No & No & Yes & Yes \\
\hline $\begin{array}{l}\text { Martin- } \\
\text { Araguz }^{45}\end{array}$ & 0 & No & Unclear & Unclear & Unclear & No & Yes & Yes & NS \\
\hline Mathew $^{46}$ & 22 & No & Unclear & Unclear & No & No & No & Yes & NS \\
\hline Mitsikostas $^{47}$ & 16 & No & Unclear & No & No & No & No & Yes & NS \\
\hline Morland $^{48}$ & 39 & No & Unclear & Unclear & Unclear & No & Unclear & Yes & NS \\
\hline $\mathrm{Nappi}^{49}$ & 0 & No & Unclear & Unclear & Unclear & Yes & Unclear & Yes & Yes \\
\hline Nelson $^{50}$ & 23 & No & Yes & Yes & No & No & Unclear & Yes & No \\
\hline Noone $^{51}$ & 52 & No & Yes & Yes & Unclear & No & Unclear & Yes & NS \\
\hline Okasha $^{52}$ & 0 & No & Yes & Unclear & Yes & Unclear & Unclear & Yes & NS \\
\hline Pfaffenrath ${ }^{53}$ & 4.1 & Yes & Unclear & Unclear & Unclear & Yes & Unclear & Yes & NS \\
\hline Rampello ${ }^{54}$ & 0 & No & Unclear & Unclear & No & Yes & Unclear & Yes & NS \\
\hline Tarosova $^{55}$ & 50 & Yes & No & Unclear & Yes & No & Unclear & Yes & NS \\
\hline Vernon $^{56}$ & 50 & No & Unclear & Unclear & Yes & No & Unclear & Yes & No \\
\hline Walker $^{57}$ & 32 & No & Unclear & Unclear & No & No & Unclear & Yes & Yes \\
\hline Ziegler $^{58}$ & 44 & Yes & Unclear & Unclear & Unclear & No & Unclear & Yes & No \\
\hline
\end{tabular}


headache (infrequent episodic $v$ frequent episodic $v$ chronic tension-type, $\mathrm{P}=0.39$ ).

The number of headaches per month among studies reporting it were reduced on average by 6.9 for tension-type headaches (95\% confidence interval -21.6 to 7.7 , three studies) and 1.4 for migraine headaches ( -3.3 to 0.5 , four studies). For both headache types this beneficial effect increased over time (tensiontype: $\beta=-0.16,95 \%$ confidence interval -0.23 to $-0.10, \mathrm{P}<0.0005$, migraine: $\beta=-0.09,-0.14$ to -0.04 $\mathrm{P}=0.001$, fig 3$)$. Therefore for every additional week of treatment with a tricyclic, the number of headaches were reduced by 0.16 standard deviations. Among these trials, a range of tricyclic doses was used, with an average dose that was $50 \%$ of the maximum for the particular tricyclic studied. The effect of doses less than $50 \%$ of the maximum dose was lower than those with higher doses (average standardised mean difference $-0.73,95 \%$ confidence interval -1.14 to $-0.32 v$ $-0.92,-1.50$ to -0.34$)$, although the difference was not significant $(\mathrm{P}=0.34)$.

Patients taking tricyclics were more likely to experience at least $50 \%$ improvement in their headaches than those taking placebo for both tension-type headaches (relative risk 1.41, 95\% confidence interval 1.02 to 1.89 , four studies, $\mathrm{I}^{2}=48.8 \%$ ) and migraine headaches $\left(1.80,1.24\right.$ to 2.62 , four studies, $\mathrm{I}^{2}=0.0 \%$, fig 4$)$. Therefore, patients treated with tricyclics had a $40 \%$ and $80 \%$ increase in the probability of having 50\% improvement in tension-type and migraine headaches, respectively. Overall, tricyclics improved the likelihood of achieving 50\% improvement in headaches (1.53, 1.16 to 2.01 , eight studies, $\mathrm{I}^{2}=26.1 \%$ ).

Tricyclics also reduced the number of doses of analgesics taken for acute headache pain for both tension-type headaches (average standardised mean difference $-1.71,95 \%$ confidence interval -3.37 to -0.05 , six studies, $\mathrm{I}^{2}=14.5 \%$ ) and migraine headaches $\left(-0.94,-1.87\right.$ to -0.02 , four studies, $\left.\mathrm{I}^{2}=32.6 \%\right)$.

Individual adverse effects of tricyclics compared with placebo were variably reported among the studies. In seven comparison arms, patients taking tricyclics were more likely to report "any" side effect than those taking placebo (relative risk 1.89, 95\% confidence interval 1.18 to $3.02, \mathrm{I}^{2}=85.9 \%$, fig 5). Among 10 individual adverse effects, only dry mouth $(2.34$, 1.63 to $\left.3.35, \mathrm{I}^{2}=43.3 \%\right)$ and drowsiness $(1.87,1.25$ to $2.70, \mathrm{I}^{2}=55.1 \%$ ) were statistically more frequent in patients receiving tricyclics than in those receiving placebo (table 3). The likelihood of withdrawing due to adverse effects, however, did not differ among 16 placebo controlled trials $\left(1.21,0.80\right.$ to $1.82, \mathrm{I}^{2}=74.1 \%$, fig 5).

Tricyclics versus selective serotonin reuptake inhibitors In a limited number of trials the effectiveness between tricyclics and selective serotonin reuptake inhibitors did not differ for tension-type headaches (average standardised mean difference $-0.80,95 \%$ confidence interval -1.63 to 0.02 , four studies, $\mathrm{I}^{2}=89.5 \%$, fig 6 ) or

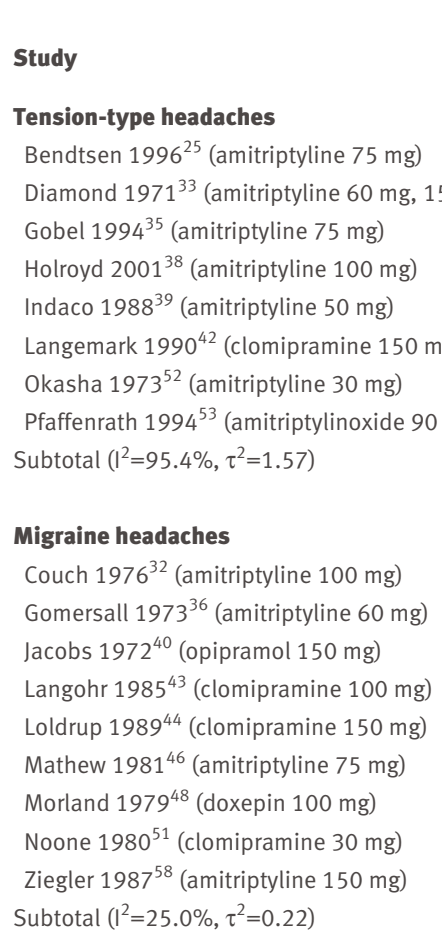

Overall $\left(\mathrm{I}^{2}=90.1 \%, \tau^{2}=0.70\right)$

\begin{tabular}{cc}
\multicolumn{2}{c}{ No/Mean (SD) } \\
\hline Treatment & Control \\
& \\
$34 / 18.6(1.6)$ & $34 / 21.7(1.3)$ \\
$39 / 0.98(1)$ & $16 / 1.56(1.4)$ \\
$24 / 7.9(6)$ & $29 / 9.6(6)$ \\
$53 / 1.8(0.2)$ & $48 / 2.6(0.2)$ \\
$15 / 8.7(2.9)$ & $16 / 18.9(4.7)$ \\
$28 / 19(20)$ & $35 / 31(20)$ \\
$20 / 0.8(3)$ & $20 / 2(3)$ \\
$133 / 208(171)$ & $64 / 208(171)$ \\
& \\
& \\
& \\
$37 / 68.5(58)$ & $36 / 104(57)$ \\
$20 / 207(132)$ & $20 / 356(132)$ \\
$13 / 2.9(1.5)$ & $12 / 4.2(1.5)$ \\
$5 / 8.85(7.7)$ & $8 / 9.65(7.7)$ \\
$84 / 273(176)$ & $87 / 351(128)$ \\
$32 / 2.28(0.36)$ & $33 / 2.72(0.31)$ \\
$14 / 462(65)$ & $14 / 512(65)$ \\
$10 / 3(2)$ & $10 / 3.5(1.2)$ \\
$30 / 429(95)$ & $30 / 511(202)$ \\
&
\end{tabular}

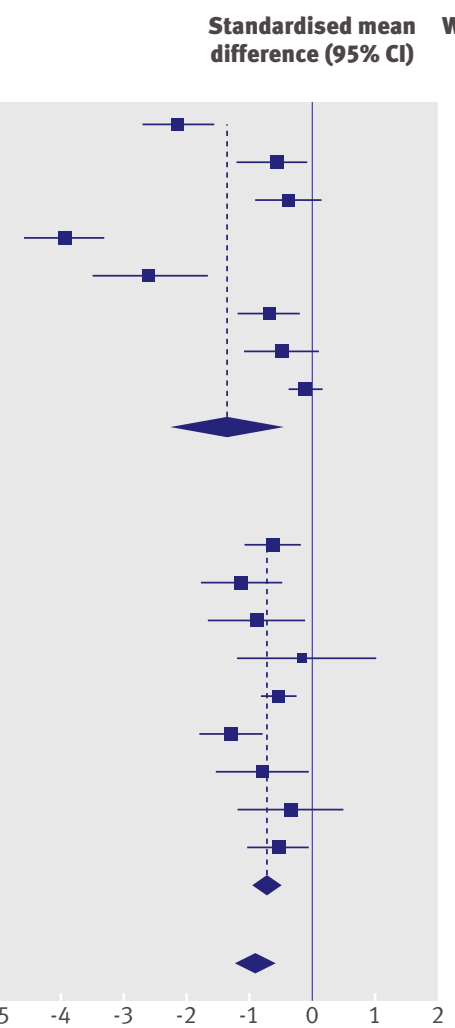

Weight (\%) Standardised mean
(random difference $(95 \% \mathrm{Cl})$ effect)

$6.02-2.13(-2.72$ to -1.53$)$

$6.04-0.52(-1.11$ to 0.07$)$

$6.15-0.28(-0.83$ to 0.26$)$

$5.82 \quad-4.00(-4.68$ to -3.32$)$

$5.06-2.59(-3.56$ to -1.62$)$

$6.22-0.60(-1.11$ to -0.09$)$

$5.96-0.40(-1.03$ to 0.23$)$

$6.60 \quad 0.00(-0.30$ to 0.30$)$

$-1.29(-2.18$ to -0.39$)$

6.30

$-0.62(-1.09$ to -0.15$)$

$-1.13(-1.80$ to -0.46$)$

$-0.87(-1.69$ to -0.04$)$

$-0.10(-1.22$ to 1.01$)$

$-0.51(-0.81$ to -0.20$)$

$-1.31(-1.85$ to -0.77$)$

$-0.77(-1.54$ to 0.00$)$

$-0.30(-1.19$ to 0.58$)$

$-0.52(-1.03$ to -0.00$)$

$-0.70(-0.93$ to -0.48$)$

$-0.96(-1.39$ to -0.53$)$

Fig 2 Effect of tricyclic antidepressants on burden of headache for tension-type and migraine headaches compared with placebo. Trials of mixed and migraine headaches are combined 

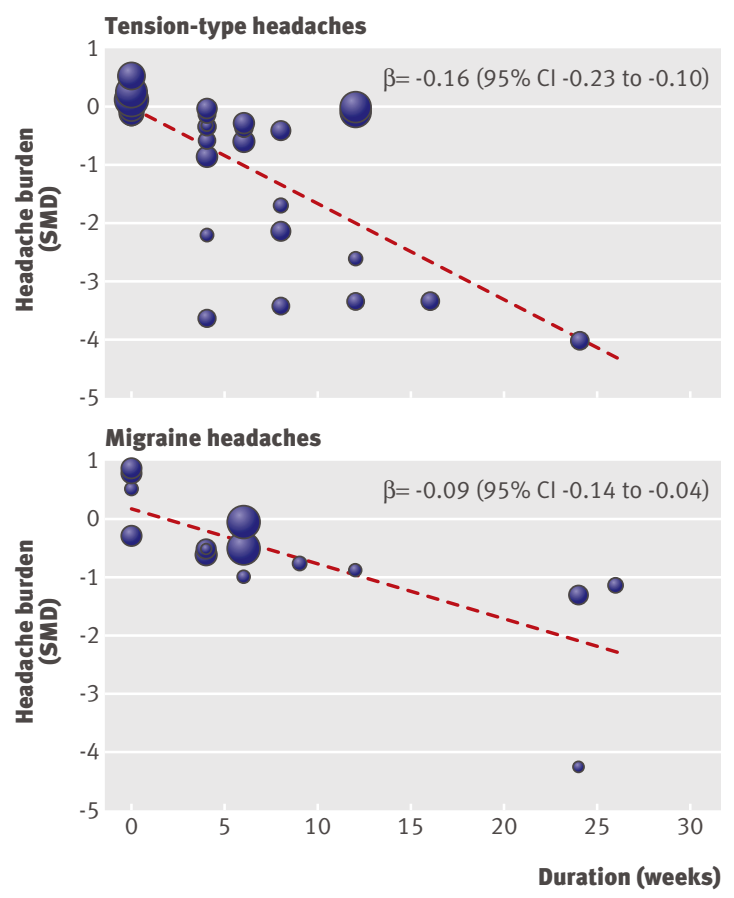

Fig 3 | Effect of tricyclic antidepressants compared with placebo over time. SMD=standardised mean difference

migraine headaches $(-0.22,-0.75$ to 0.31 , four studies, $\mathrm{I}^{2}=89.5 \%$ ). Seven of these trials used amitriptyline and one used desipramine. The doses used were relatively low: the maximum dose of amitriptyline averaged $50 \mathrm{mg}$, ranging from $25 \mathrm{mg}$ to $75 \mathrm{mg}$; the dose of desipramine was $150 \mathrm{mg}$. Despite low doses, tricyclics were more likely than selective serotonin reuptake inhibitors to produce at least $50 \%$ improvement in tension-type headaches (relative risk 1.73, 95\% confidence interval 1.34 to $2.22, \mathrm{I}^{2}=0.0 \%$, three studies, fig 7 ), and migraine headaches $(1.72,1.15$ to 2.55 , $\mathrm{I}^{2}=9.2 \%$, three studies). Tricyclic arms had higher rates of several adverse effects such as drowsiness and dry mouth (table 3), although they did not have higher withdrawal rates for tension-type headaches $(1.05,0.92$ to 1.19 , two studies, $\mathrm{I}^{2}=0.0 \%$ ) or migraine headaches (2.0, 0.90 to 4.47 , three studies, $\left.\mathrm{I}^{2}=0.0 \%\right)$.

Among three trials, tricyclic arms were more likely to have "any" side effect than the selective serotonin reuptake inhibitor arms $\left(2.25,1.52\right.$ to $3.32, \mathrm{I}^{2}=$ $0.0 \%){ }^{252857}$ Specific side effects more common among tricyclics included abdominal distress (2.34, 1.31 to 4.95 , four studies, $\mathrm{I}^{2}=6.6 \%$ ), dizziness $(2.52$, 0.77 to 8.23 , five studies, $\mathrm{I}^{2}=63.0 \%$ ), and dry mouth (4.88, 2.26 to 10.52 , five studies, $\mathrm{I}^{2}=67.8 \%$ ), although the risk of withdrawing from the study did not differ (1.16, 0.81 to 2.97 , five studies, $\mathrm{I}^{2}=19.0 \%$ ).

\section{Tricyclics versus other drugs}

Few studies compared tricyclics with other modalities. No studies compared $\beta$ blockers and tricyclics for tension-type headaches; among three studies of migraine headaches, tricyclics and $\beta$ blockers did not differ in reduction of number of headaches or attacks (average standardised mean difference $0.90,95 \%$ confidence interval -0.38 to $2.18, \mathrm{I}^{2}=93.8 \%$ ) and in two studies did not differ in the likelihood of experiencing at least $50 \%$ reduction in the number of headaches (relative risk $0.78,95 \%$ confidence interval 0.42 to 1.44 , $\mathrm{I}^{2}=0.0 \%$. Three of the studies used amitriptyline and one used clomipramine; the average dose for both was $100 \mathrm{mg}$.

Two studies of tension-type headaches compared tricyclics with buspirone (amitriptyline $50 \mathrm{mg}$ ) and found no differences (buspirone: average standardised mean difference $-1.01,95 \%$ confidence interval -1.83 to $-0.18, \mathrm{I}^{2}=0.00 \%$, table 4 ). Two studies compared tricyclics (amitriptyline $25 \mathrm{mg}$, clomipramine $150 \mathrm{mg}$ ) with tetracyclics and found no difference (average standardised mean difference $-0.21,-0.65$ to 0.22 ,

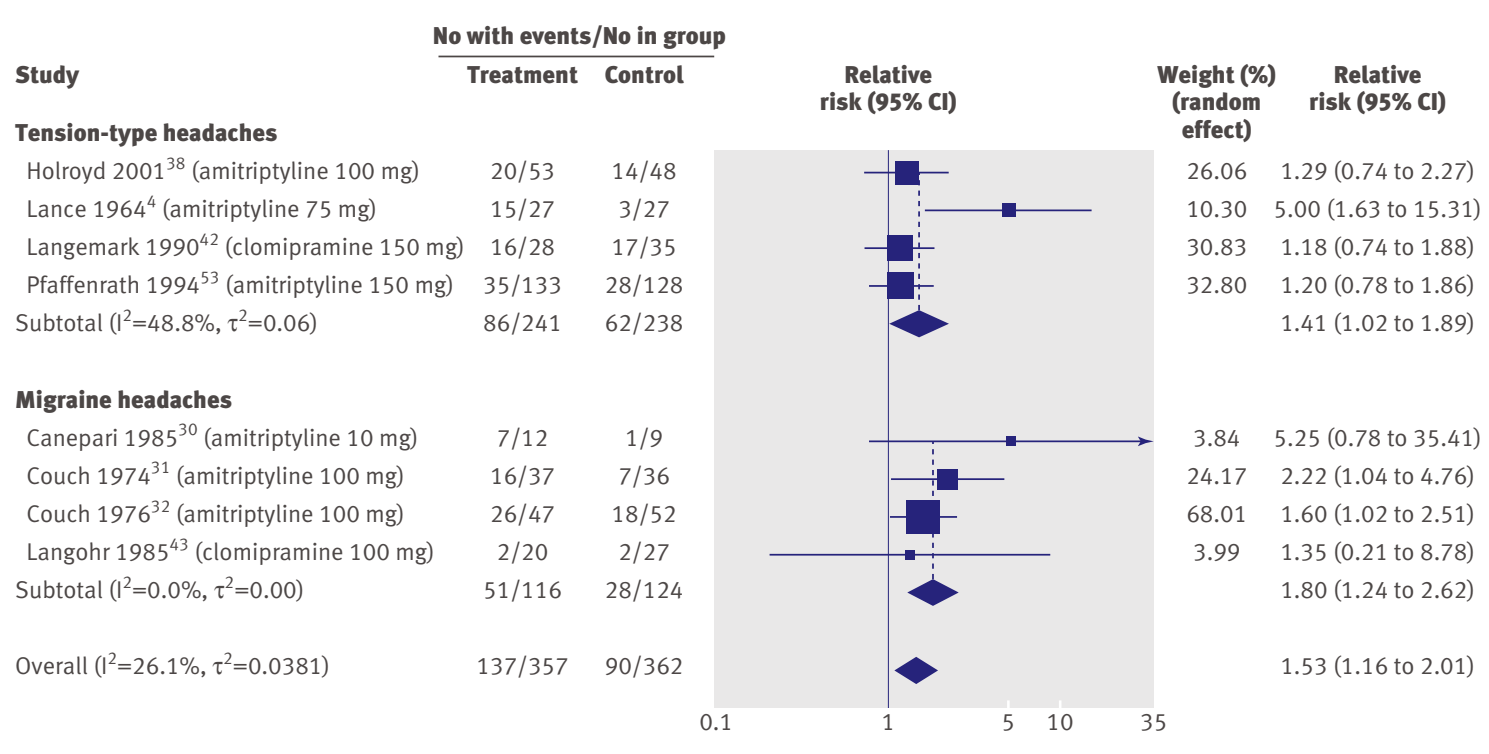

Fig 4 Likelihood of experiencing at least $50 \%$ reduction in tension-type and migraine headaches compared with placebo. Trials of mixed and migraine headaches are combined 




Fig 5 | Likelihood of adverse effects between tricyclic antidepressants and placebo

$\mathrm{I}^{2}=32.8 \%$ ). A single study of tension-type headache found no difference between low dose amitriptyline $(25 \mathrm{mg})$ and the serotonin antagonist ritanserin $(-0.49,-1.70$ to 0.71$)$. One study found that amitriptyline $(75 \mathrm{mg})$ was better than timed release dihydroergotamine for migraines $(-6.57,-8.44$ to -4.71$)$. In addition, two trials compared amitriptyline (maximum doses $100 \mathrm{mg}$ and $150 \mathrm{mg}$ ) with the anticonvulsant topiramate $\left(0.05,-0.32\right.$ to $0.33, \mathrm{I}^{2}=29.2 \%$, table 4$)$ and one (amitriptyline $75 \mathrm{mg}$ ) with the calcium channel blocker flunarazine (relative risk 1.60, 95\% confidence interval 0.83 to 3.10 , table 5) in migraine headaches, and found no differences.

\section{Tricyclics versus non-drug treatments}

No differences were found between spinal manipulation and tricyclics in two studies of tension-type headaches (average standardised mean difference -0.25 $95 \%$ confidence interval -0.59 to $0.09, \mathrm{I}^{2}=0.0 \%$, table 4$)$ and one study of migraine headaches $(-0.31,-0.69$ to

Table 3|Relative risk of adverse effects among tricyclic antidepressants compared with placebo or selective serotonin reuptake inhibitors

\begin{tabular}{|c|c|c|c|c|c|c|}
\hline Adverse effect & Placebo & No of studies & $\begin{array}{c}\text { Heterogeneity (I2) } \\
\%\end{array}$ & $\begin{array}{l}\text { Selective serotonin } \\
\text { reuptake inhibitors }\end{array}$ & No of studies & Heterogeneity (I2) \% \\
\hline Anxiety or irritability & $0.44(0.15$ to 1.31$)$ & 2 & 0.0 & $0.67(0.23$ to 1.91$)$ & 2 & 0.0 \\
\hline Blurred vision & $1.05(0.56$ to 1.97$)$ & 4 & 0.0 & $\mathrm{NR}$ & & \\
\hline Constipation or abdominal pain & 1.07 (0.61 to 1.86$)$ & 7 & 6.4 & 2.34 (1.31 to 4.95$)$ & 4 & 6.6 \\
\hline Diaphoresis & 1.09 (0.38 to 3.08$)$ & 3 & 73.0 & NR & NR & NR \\
\hline Dizziness & $1.20(0.77$ to 1.86$)$ & 7 & 0.0 & $2.52(0.77$ to 8.23$)$ & 5 & 63.0 \\
\hline Drowsiness & 1.87 (1.25 to 2.70$)$ & 10 & 55.1 & 2.64 (1.84 to 3.78$)$ & 6 & 17.0 \\
\hline Dry mouth & 2.34 (1.63 to 3.35$)$ & 10 & 43.3 & $4.88(2.26$ to 10.52$)$ & 5 & 67.8 \\
\hline Nausea or vomiting & 1.18 (0.42 to 3.31$)$ & 4 & 22.8 & $0.62(0.26$ to 1.48$)$ & 4 & 57.8 \\
\hline Sleep disturbance & $0.64(0.37$ to 1.09$)$ & 5 & 0.0 & $0.52(0.17$ to 1.58$)$ & 3 & 0.0 \\
\hline Weight gain & 1.69 (0.74 to 3.88$)$ & 6 & 49.2 & 7.45 (0.99 to 56.37$)$ & 4 & 72.0 \\
\hline
\end{tabular}

NR=not reported. 


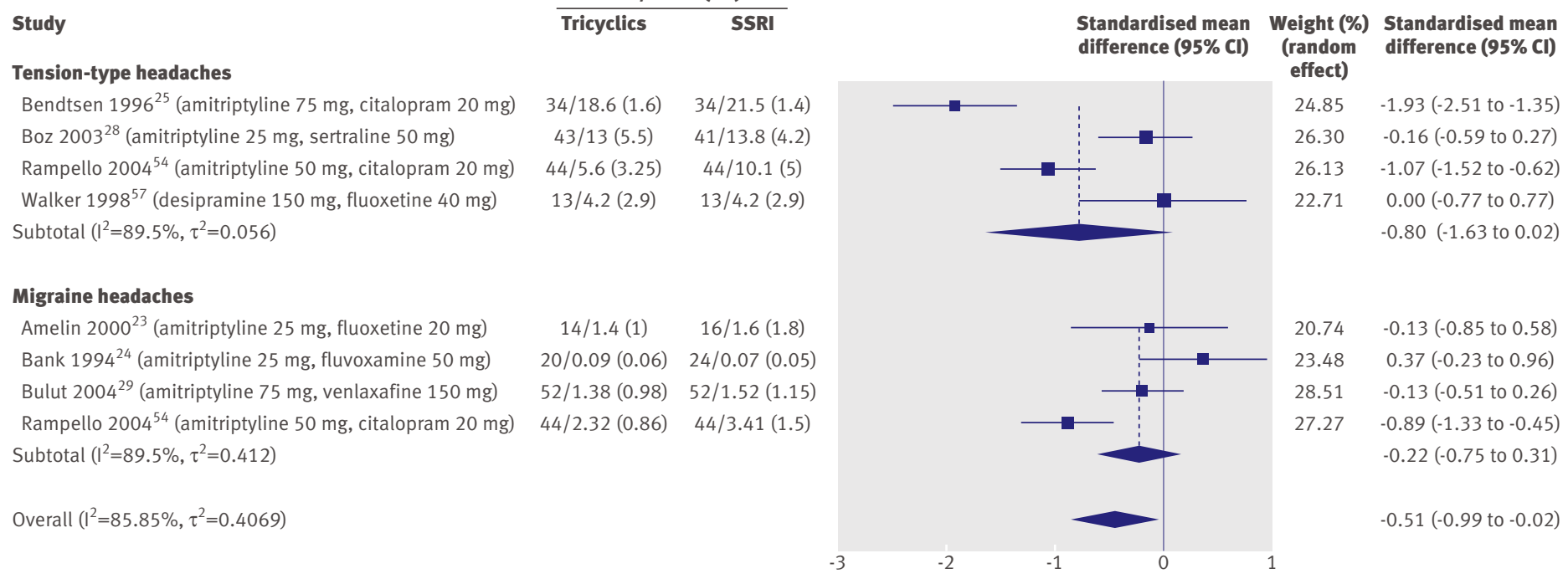

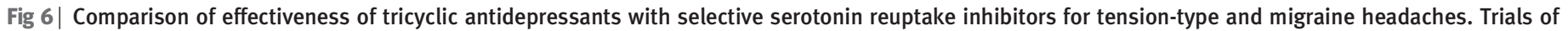
mixed and migraine headaches are combined. SSRI=selective serotonin reuptake inhibitor

0.08). All three studies used amitriptyline, with maximum doses of $25 \mathrm{mg}, 30 \mathrm{mg}$, and $100 \mathrm{mg}$. Tricyclics were compared with cognitive behavioural therapy in one study of tension-type headaches and in two studies of migraine headaches, with no differences (tables 4 and 5). All three used amitriptyline at a dose of $75 \mathrm{mg}$.

\section{Assessment of bias and sensitivity analyses}

The Cochrane criteria seemed to show considerable risk of bias (table 2). On analysis of the components, we found no difference in effects between studies meeting or not meeting any Cochrane criteria for bias. We also found no effect of industry sponsorship or assessment of adherence on outcomes. We found evidence of publication bias for continuous outcomes $(\mathrm{P}=0.06)$ and dichotomous outcomes $(\mathrm{P}=0.04)$. Estimates based on Gleser's method suggested that up to 10 unpublished trials reporting continuous outcomes and four unpublished trials reporting dichotomous outcomes could exist. Rosenthal's fail safe suggests that 1467 unpublished trials with continuous outcomes and 691 unpublished trials with dichotomous outcomes with no effect would be required to reduce our effect to clinical insignificance (effect size $<0.3$ ). The meta-trim test did not affect the continuous outcome summary effect, but reduced the dichotomous outcome from a relative risk of $1.53(95 \%$ confidence interval 1.16 to 2.01 ) to one of 1.50 (1.13 to 1.99). Metaregression found no effect of any other variables abstracted on summary outcomes, with the exception of study duration. Duration explained $32.4 \%$ of the heterogeneity for continuous outcomes and $27.3 \%$ of the variance for dichotomous outcomes.

\section{DISCUSSION}

We found that tricyclic antidepressants substantially reduced the pain from both migraine and tension-

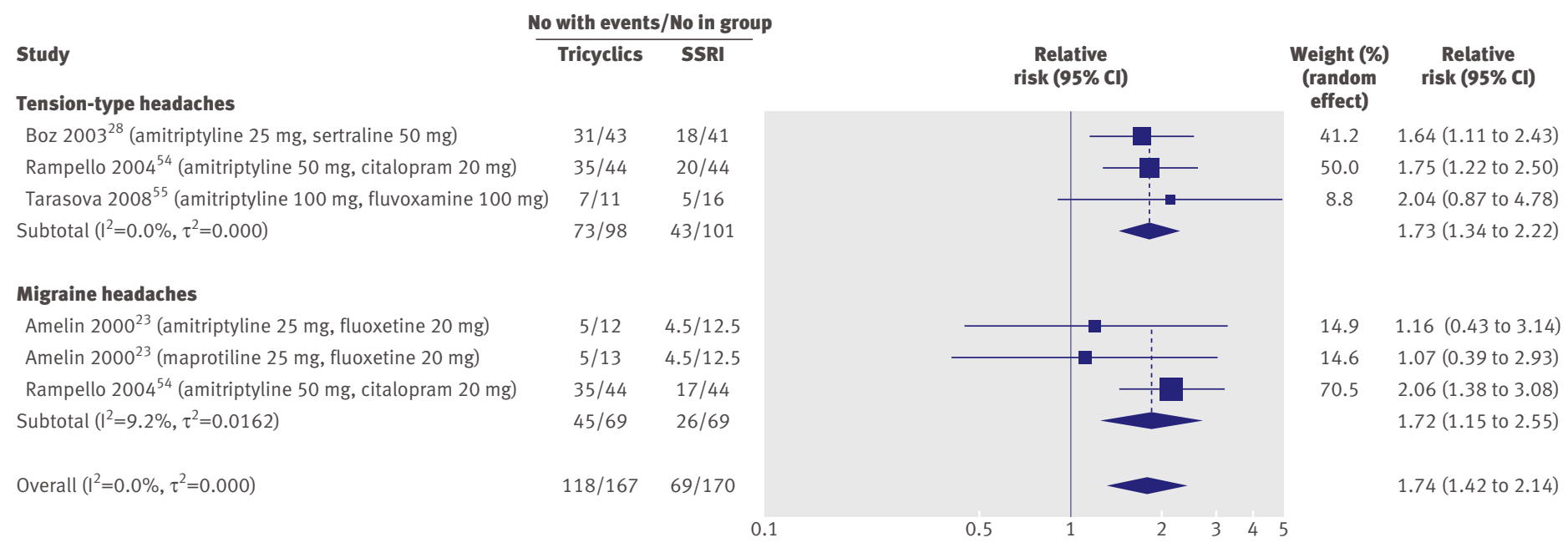

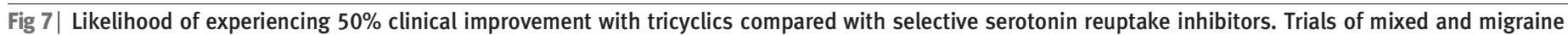
headaches are combined. SSRI=selective serotonin reuptake inhibitor 
Table 4 |Average standardised mean differences between tricyclic antidepressants and non-placebo treatments for tension-type and migraine headaches

\begin{tabular}{|c|c|c|c|c|c|c|c|c|}
\hline \multirow[b]{2}{*}{ Comparison class } & \multicolumn{4}{|c|}{ Tension-type headache } & \multicolumn{4}{|c|}{ Migraine headache } \\
\hline & $\begin{array}{c}\text { Drugs } \\
\text { compared }\end{array}$ & Average SMD $(95 \% \mathrm{Cl})$ & $\begin{array}{c}\text { No of } \\
\text { studies }\end{array}$ & $\begin{array}{c}\text { Heteroge- } \\
\text { neity (I2)\% }\end{array}$ & $\begin{array}{c}\text { Drugs } \\
\text { compared }\end{array}$ & Average SMD $(95 \% \mathrm{Cl})$ & $\begin{array}{c}\text { No of } \\
\text { studies }\end{array}$ & $\begin{array}{l}\text { Heterogeneity } \\
\text { (I2) } \%\end{array}$ \\
\hline Anticonvulsant & - & - & - & - & Topiramate & $0.05(-0.32$ to 0.33$)$ & 2 & 29.2 \\
\hline$\beta$ blocker & - & - & - & - & $\begin{array}{l}\text { Propranolol } \\
(\times 3) \text {, } \\
\text { metoprolol }\end{array}$ & $0.90(-0.38$ to 2.18$)$ & 4 & 93.8 \\
\hline Biofeedback & - & - & - & - & - & $-0.93(-2.76$ to 0.91$)$ & 2 & 95.5 \\
\hline Buspirone & Buspirone & $-1.01(-1.83$ to -0.18$)$ & 2 & 0 & - & - & - & - \\
\hline Cognitive behavioural therapy & - & $0.59(-0.08$ to 1.26$)$ & 1 & - & - & - & - & - \\
\hline Ergotamine & - & - & - & - & - & $-6.57(-8.40$ to -4.71$)$ & 1 & - \\
\hline Serotonin antagonist & Ritanserin & $-0.49(-1.70$ to 0.71$)$ & 1 & - & - & - & - & - \\
\hline Spinal manipulation & - & $-0.25(-0.59$ to 0.09$)$ & 2 & 0 & - & $-0.31(-0.69$ to 0.08$)$ & 1 & - \\
\hline Tetracyclic & $\begin{array}{l}\text { Mianserin, } \\
\text { mirtazapine }\end{array}$ & $-0.212(-0.65$ to 0.22$)$ & 2 & 32.8 & Maprotiline & $-0.15(-0.87$ to 0.57$)$ & 1 & - \\
\hline
\end{tabular}

type headaches. Patients treated prophylactically with tricyclics experienced about 1 standard deviation of improvement in headache burden, a clinically large effect. Patients with tension-type or migraine headaches were $40-70 \%$ more likely to report at least a $50 \%$ improvement in headaches, and patients taking tricyclics for tension-type headaches used fewer analgesics. Moreover, the effect seems to increase over time; patients in the first month of treatment had less improvement than those treated for six months. Some adverse effects, particularly dry mouth and drowsiness, were more likely with tricyclics, although this did not result in significantly greater dropout rates, implying a tolerable side effect profile given the benefit. Tricyclics were more effective than selective serotonin reuptake inhibitors in achieving 50\% reduction in headaches in a small number of comparative studies. People taking tricyclics were more likely to experience dry mouth, drowsiness, and abdominal distress than those taking selective serotonin reuptake inhibitors, but were not more likely to withdraw from the study.

In a previous meta-analysis, we found that antidepressants reduced headache burden compared with placebo. ${ }^{7}$ This study expands that work in several important ways. Previously we included only English language articles and only compared antidepressants with placebo. This study has a greater number of trials and includes comparisons with modalities other than placebo. This allowed us to show that longer treatment improved the efficacy of tricyclics. We were also able to show that tricyclics are superior to selective serotonin reuptake inhibitors. A recent Cochrane review found that selective serotonin reuptake inhibitors were no more effective than placebo for tension-type headaches. They found some indicators that selective serotonin reuptake inhibitors were less effective than tricyclics for migraines (likelihood of taking $>5$ analgesic doses per month, number of hours per day with headache). ${ }^{60}$ They concluded that tricyclics were favoured over selective serotonin reuptake inhibitors, consistent with the findings reported here.

It is difficult to reach firm conclusions on the relative efficacy of tricyclics compared with other treatment modalities. There were relatively few studies and most were underpowered to assess clinical equivalence.

Tricyclics seem equally effective for tension-type, migraine, and mixed headaches. This is useful information for clinicians as differentiating these headache types may not always be straightforward, especially in patients with frequent headaches. While this similar efficacy lends support to the clinical practice of using the same treatment approaches for recurrent tension, mixed and migraine headache, it does not address important underlying theoretical issues. A similarity in the magnitude of effect does not prove that the mechanisms underlying these headache types are the same, that they converge on some final common pathway, or that the different classes of antidepressants act through some common mechanism. Neither the

Table 5 | Effect of tricyclic antidepressants compared with non-placebo treatments on reduction in headache burden by $50 \%$

\begin{tabular}{|c|c|c|c|c|}
\hline \multirow[b]{3}{*}{ Comparison class } & \multicolumn{2}{|c|}{ Tension-type headache } & \multicolumn{2}{|c|}{ Migraine headache } \\
\hline & & Relative risk (95\% & & \\
\hline & Drugs & Cl) & Drugs & Relative risk $(95 \% \mathrm{Cl})$ \\
\hline Anticonvulsant & - & - & Topirimate & $0.82(0.61 \text { to } 1.09)^{*}$ \\
\hline$\beta$ blocker & - & - & $\begin{array}{l}\text { Metoprolol, } \\
\text { propranolol }\end{array}$ & $0.78(0.42 \text { to } 1.44)^{\star}$ \\
\hline Calcium channel blocker & - & - & Flunarazine & $1.60(0.83 \text { to } 3.10)^{\star}$ \\
\hline Cognitive behavioural therapy & - & $1.09(0.65 \text { to } 1.82)^{\star}$ & - & $0.48(0.14 \text { to } 1.57)^{\star}$ \\
\hline Tetracyclic & Mianserin & $1.07(0.67 \text { to } 1.71)^{*}$ & - & - \\
\hline
\end{tabular}


studies we examined, nor this study, were designed to resolve issues of nosology and mechanism.

We also found evidence that the benefit of tricyclics for headaches increased with longer study duration. This is consistent with clinical practice and treatment recommendations suggesting that tricyclics need to be taken for several months before reaching any conclusion on effectiveness for any given patient. The scarcity of studies with longer duration is problematic and casts suspicion on the reliability of any conclusions about the long term efficacy of tricyclics.

\section{Strengths and weaknesses of the study}

This study's strengths include its methodological rigor and the care taken in study design, data abstraction, and data analysis. However, our meta-analysis has several limitations. Firstly, one of the most important questions is whether tricyclics are better than other commonly used prophylactic treatments, such as $\beta$ blockers or anticonvulsants. Unfortunately, few trials compare tricyclics head to head against other modalities and many of these comparisons seem to have been designed to put tricyclics at a disadvantage - for example, the average dose of tricyclics used in the comparison with selective serotonin reuptake inhibitors is lower than most clinicians would use in treating headaches. More comparative effectiveness trials are needed.

Secondly, because of the large number of analyses, caution is required in interpreting our finding of an association between treatment duration and efficacy. This analysis should be viewed as exploratory rather than definitive. Moreover, most trials were relatively short, particularly since headaches tend to be a chronic problem. The average duration of the trials in our study was 11 weeks and the longest only 26 weeks; consequently our timeline was constrained to four to 26 weeks. This finding is consistent with clinical experience. Many clinicians encourage their patients to take tricyclics for several months, during which the dose is slowly increased, before deciding to try a different prophylactic agent. This also suggests that treatment trials of longer duration are urgently needed.

Thirdly, it is impossible to differentiate whether treatment effect was independent of depression. The relation between depression and physical symptoms is well described. Patients with depression have more physical symptoms and report those symptoms as more severe and disabling than patients without depression. ${ }^{6162}$ Depressed patients experience improvement in somatic problems when underlying depression is successfully treated. ${ }^{63}$ Patients with depression have also been found to have more headaches, ${ }^{64}$ although others have argued that this is a result of chronic underlying pain rather than the cause of it. ${ }^{65}$ The effects of tricyclic antidepressants on pain syndromes that are considered unrelated to depression, such as neuropathy, have led investigators to suggest a direct analgesic effect. ${ }^{667}$ Serotonin has been found to be an active neurotransmitter in the central modulation of pain, ${ }^{68}$ so there is a possible effect on pain mechanisms other than through depression. Given the short duration and low doses of tricyclics used for headaches compared with those needed to treat depression, it seems unlikely that the mechanism for the success of headache treatment in these trials is entirely due to the treatment of depression.

A fourth limitation is the lack of reporting on concomitant use of direct, "as needed," drugs. Although most of the studies included patient diaries on the use of analgesics during or before the study period, only four placebo controlled studies and two comparisons with selective serotonin reuptake inhibitors provide any results, all showing insignificantly greater reduction in analgesic use among patients receiving tricyclics. When data are combined across headache types, there is evidence of reduction in analgesic use. However, it is possible that other trials collected but did not report their data; there may be a bias against presenting data suggesting less treatment benefit. Reducing the need for patient use of analgesics is an important goal of prophylactic therapy in general, and has the additional benefit of helping to prevent "rebound" or drug overuse headaches.

A fifth limitation is that it is impossible to tell from our data what the optimal dose is for preventing headaches. Meta-analysis is limited by the problem of aggregate data. Optimal dosing is a question that could be answered only with the availability of patient level data, given the relatively large number of trials that have been carried out and the fact that nearly all trials included titration of tricyclics.

Sixthly, study quality was low in general. Few studies did an intention to treat analysis. This is particularly worrisome since some trials experienced up to $50 \%$ losses to follow-up. It is easy to imagine that patients who either had tremendous adverse effects or had lack of improvement would be particularly likely to drop out. We found no difference in effect estimates between those who did and did not do an intention to treat analysis, but our sample was relatively small.

Finally, heterogeneity in our results was considerable, particularly among people with tension-type headaches. Study duration explained some, but not all, of the variance. In addition, there was evidence of small study effects on our results, also known as publication bias. Tests to estimate the number of unpublished trials to calculate the number of unpublished trials necessary to obviate results and tests to "fill in" missing studies have been criticised for lack of underlying theory, poor performance in simulations, and a tendency to overestimate effects, particularly in the face of heterogeneity. ${ }^{69}$ Since small, negative trials are less likely to be published, such missing trials would generally decrease the efficacy estimate. It is important to keep in mind, however, two important potential sources of confounding. Firstly, the treatment effect is real. Effective treatments would also seem to have evidence of publication bias. Secondly, tests for publication bias tend to overestimate risk of bias in the face of heterogeneity. ${ }^{69}$ It is possible, indeed probable, that 


\section{WHAT IS ALREADY KNOWN ON THIS TOPIC}

Tricyclic antidepressants are effective in treating tension-type and migraine headaches

Selective serotonin reuptake inhibitors are not more effective in the treatment of migraine headaches than tension-type headaches

\section{WHAT THIS STUDY ADDS}

Tricyclics are more effective over time

Tricyclics are more effective than selective serotonin reuptake inhibitors for both migraine and tension-type headaches

The number of trials comparing tricyclics with other treatment modalities is inadequate

unpublished trials exist. The effect we report is therefore possibly an overestimation.

Despite these limitations, our meta-analysis shows the efficacy of tricyclics in people both with migraine headache and with tension-type headache. The benefit seems moderate to large in magnitude. Helpful studies would be those that determine whether particular subgroups of patients are more likely to respond to treatment with tricyclics, such as depressed patients or those with high use of analgesics. It would also be useful to better define more effective treatment regimens, in terms of target doses, treatment duration, or interaction with direct analgesics. Trials directly comparing treatment between tricyclics and other prophylactic regimens are needed.

This paper is based on a Cochrane review first published in the Cochrane Library (www.thecochranelibrary.com for information). Cochrane reviews are regularly updated as new evidence emerges and in response to comments and criticisms. The Cochrane Library should be consulted for the most recent version of the review. The results of a Cochrane review can be interpreted differently, depending on people's perspectives and circumstances. Please consider the conclusions presented carefully. They are the opinions of review authors, and are not necessarily shared by the Cochrane Collaboration

Contributors: JL) had full access to the data in the study and takes responsibility for the integrity of the data and the accuracy of the data analysis. He is guarantor. All authors were involved in the study design and data collection, contributed to the writing and revision of the manuscript, and are able to take responsibility for its content. All authors were present at idea inception and were assigned various specific tasks in the process of creating this manuscript. All tasks were done in duplicate: LS and EB retrieved the articles, LS and MD selected the articles, WS and KJD abstracted the data, and JLI and DB rated the articles for quality. JLJ and LS carried out the analysis. All the opinions in this article are those of the authors and should not be construed to reflect, in any way, those of the Department of the Army, Navy, the Department of Defense or the Department of Veterans Affairs.

Funding: This study received no external funding. Our institutional review board reviewed and approved this manuscript for publication.

Competing interests: All authors have completed the Unified Competing Interest form at (www.icmje.org/coi_disclosure.pdf (available on request from the corresponding author) and declare that (1) they have no relationships with companies that might have an interest in the submitted work; (2) their spouses, partners, or children have no financial relationships that may be relevant to the submitted work; and (3) no authors have any non-financial interests that may be relevant to the submitted work.

Ethical approval: Not required.

Data sharing: No additional data available.

1 Wang SJ. Epidemiology of migraine and other types of headache in Asia. Curr Neurol Neurosci Rep 2003;3:104-8.

2 Rasmussen BK. Epidemiology of headache. Cephalalgia 2001;21:774-7.
$3 \mathrm{Hu}$ XH, Markson LE, Lipton RB, Stewart WF, Berger ML. Burden of migraine in the United States: disability and economic costs. Arch Intern Med 1999;159:813-8.

4 Lance JW, Curan DA. Treatment of chronic tension headache. Lancet 1964;1:1236-9.

5 Lipton RB, Bigal ME, Diamond M, Freitag F, Reed ML, Stewart WF. Migraine prevalence, disease burden, and the need for preventive therapy. Neurology 2007;68:343-9.

6 Rapaport AM. Recurrent migraine: cost-effective care. Neurology 1994:44(suppl 3):25-8S

7 Tomkins GE, Jackson JL, O’Malley PG, Balden E, Santoro JE. Treatment of chronic headache with antidepressants: a meta-analysis. Am J Med 2001;111:54-63.

8 Headache Classification Subcommittee of the International Headache Society. The international classification of headache disorders. 2nd ed. Cephalalgia 2004;24(suppl 1):1-169S.

9 Ad Hoc Committee on the Classification of Headache of the Nationa Institute of Neurological Diseases and Blindness. Classification of headache. JAMA 1962;179:717-8.

10 Headache Classification Committee of the International Headache Society. Classification and diagnostic criteria for headache disorders, cranial neuralgias and facial pain. Cephalalgia 1988;8(suppl 7):1-96S.

11 Follmann D, Elliott P, Suh I, Cutler J. Variance imputation for overviews of clinical trials with continuous response. J Clin Epidemio 1992;45:769-73.

12 Alderson P, Green S, Higgins JPT. Assessment of study quality. In: Alderson P, Green S, Higgins JPT, eds. Cochrane reviewers' handbook 4.2.2. Wiley, 2004

13 Kazis LE, Anderson JJ, Meenan RF. Effect sizes for interpreting changes in health status. Med Care 1989;27(suppl 3):178-89S.

14 DerSimonian R, Laird N. Meta-analysis in clinical trials. Control Clin Trials 1986;7:177-88.

15 Galbraith RF. A note on graphical presentation of estimated odds ratios from several clinical trials. Stat Med 1988;7:889-94.

16 Higgins JP, Thompson SG, Deeks JJ, Altman DG. Measuring inconsistency in meta-analyses. BMJ 2003;327:557-60.

17 Peters JL, Sutton AJ, Jones DR, Abrams KR, Rushton L. Comparison of two methods to detect publication bias in meta-analysis. JAMA 2006;295:676-80.

18 Egger M, Davey Smith G, Schneider M, Minder C. Bias in metaanalysis detected by a simple, graphical test. BMJ 1997;315:629-34.

19 Gleser LJ, Olkin I. Models for estimating the number of unpublished studies. Stat Med 1996;15:2493-507.

20 Rosenthal R. The file drawer problem and tolerance for null results. Psychol Bull 1979;86:638-41.

21 Duval S, Tweedie R. Trim and fill: a simple funnel-plot-based method of testing and adjusting for publication bias in meta-analysis. Biometrics 2000;56:55-463.

22 Sharp S. Meta-analysis regression. Stata Tech Bull 1998;42:16-22.

23 Amelin AV, Skoromets AA, Korenko LA, Tumelevich BC, Gonchar MA. [A comparative efficiency of amitriptyline, fluoxetine and maprotiline in prevention of migraine in attack-free period.] Zhurnal Nevropatoliogii I Psikhiatrii Imeni S S Korsakova 2000;100:20-3.

24 Bank J. A comparative study of amitriptyline and fluvoxamine in migraine prophylaxis. Headache 1994:34:476-8.

25 Bendtsen L, Jensen R, Olesen J. A non-selective (amitriptyline), but not a selective (citalopram), serotonin reuptake inhibitor is effective in the prophylactic treatment of chronic tension-type headache. J Neurol Neurosurg Psychiatr 1996;61:285-90.

26 Boline PD, Kassak K, Bronfort G, Nelson C, Anderson AV. Spinal manipulation vs amitriptyline for the treatment of chronic tensiontype headaches: a randomized clinical trial. J Manipulative Physiol Ther 1995;18:148-54

27 Bonuso S, Di Stasio E, Barone P, Steardo L. Timed-release dihydroergotamine in the prophylaxis of mixed headache. A study versus amitriptyline. Cephalalgia 1983;3(suppl 1):175-8.

28 Boz C, Altunayoglu V, Velioglu S, Ozmenoglu M. Sertraline versus amitriptyline in the prophylactic therapy of non-depressed chronic tension-type headache patients. J Headache Pain 2003;4:72-8.

29 Bulut S, Berilgen MS, Baran A, Tekatas A, Atmaca M, Mungen B. Venlafaxine versus amitriptyline in the prophylactic treatment of migraine: randomized, double-blind, crossover study. Clin Neurol Neurosurg 2004;107:44-8.

30 Canepari C, Riva M, Erminio F. [Effectiveness of flunarizine in chronic headache. Double-blind study of placebo and amitriptyline.] $\mathrm{La}$ Clinica Terapeutica 1985;115:463-8.

31 Couch JR, Ziegler DK, Hassanein RS. Evaluation of amitriptyline in migraine prophylaxis. Trans Am Neurol Assoc 1974;99:94-9.

32 Couch JR, Hassanein RS. Migraine and depression: effect of amitriptyline prophylaxis. Trans Am Neurol Assoc 1976;101:234-7.

33 Diamond S, Baltes BJ. Chronic tension headache - treated with amitriptyline-a double-blind study. Headache 1971;11:110-6.

34 Dodick DW, Freitag F, Banks J, Saper J, Xiang J, Rupnow M, et al. Topiramate versus amitriptyline in migraine prevention: a 26-week, 
multicenter, randomized, double-blind, double-dummy, parallelgroup noninferiority trial in adult migraineurs. Clin Ther 2009;31:542-59.

35 Gobel H, Hamouz V, Hansen C, Heininger K, Hirsch S, Lindner V, et al. Chronic tension-type headache: amitriptyline reduces clinical headache-duration and experimental pain sensitivity but does not alter pericranial muscle activity readings. Pain 1994;59:241-9.

36 Gomersall JD, Stuart A. Amitriptyline in migraine prophylaxis. Changes in pattern of attacks during a controlled clinical trial. J Neurol Neurosurg Psychiatr 1973;36:684-90.

37 Holroyd KA, Nash JM, Pingel JD, Cordingley GE, Jerome A. A comparison of pharmacological (amitriptyline $\mathrm{HCL}$ ) and nonpharmacological (cognitive-behavioral) therapies for chronic tension headaches. J Consult Clin Psychol 1991;59:387-93.

38 Holroyd KA, O’Donnell FJ, Stensland M, Lipchik GL, Cordingley GE, Carlson BW. Management of chronic tension-type headache with tricyclic antidepressant medication, stress management therapy, and their combination: a randomized controlled trial. J Am Med Asso 2001;285:2208-15.

39 Indaco A, Carrieri PB. Amitriptyline in the treatment of headache in patients with Parkinson's disease: a double-blind placebocontrolled study. Neurology 1988;38:1720-2.

40 Jacobs H. A trial of opipramol in the treatment of migraine. J Neurol Neurosurg Psychiatr 1972;35:500-4

41 Keskinbora K, Aydinli I. A double-blind randomized controlled trial of topiramate and amitriptyline either alone or in combination for the prevention of migraine. Clin Neurol Neurosurg 2008;110:979-84.

42 Langemark M, Loldrup D, Bech P, Olesen J. Clomipramine and mianserin in the treatment of chronic tension headache. A doubleblind, controlled study. Headache 1990;30:118-21.

43 Langohr HD, Gerber WD, Koletzki E, Mayer K, Schroth G. Clomipramine and metoprolol in migraine prophylaxis-a doubleblind crossover study. Headache 1985;25:107-13.

44 Loldrup D, Langemark M, Hansen HJ, Olesen J, Bech P. Clomipramine and mianserin in chronic idiopathic pain syndrome. A placebo controlled study. Psychopharmacology 1989;99:1-7.

45 Martin-Araguz A, Bustamante-Martinez C, Pedro-Pijoan JM. [Treatment of chronic tension type headache with mirtazapine and amitriptyline]. Revista de Neurologia 2003;37:101-5.

46 Mathew NT. Prophylaxis of migraine and mixed headache. A randomized controlled study. Headache 1981;21:105-9.

47 Mitsikostas DD, Gatzonis S, Thomas A, Ilias A. Buspirone vs amitriptyline in the treatment of chronic tension-type headache. Acta Neurol Scand 1997;96:247-51.

48 Morland TJ, Storli OV, Mogstad TE. Doxepin in the prophylactic treatment of mixed "vascular" and tension headache. Headache 1979;19:382-3

49 Nappi G, Sandrini G, Granella F, Ruiz L, Cerutti G, Facchinetti F, et al. A new 5-HT2 antagonist (ritanserin) in the treatment of chronic headache with depression. A double-blind study vs amitriptyline. Headache 1990;30:439-44.

50 Nelson CF, Bronfort G, Evans R, Boline P, Goldsmith C, Anderson AV. The efficacy of spinal manipulation, amitriptyline and the combination of both therapies for the prophylaxis of migraine headache. J Manipulative Physiol Ther 1998;21:511-9.

51 Noone JF. Clomipramine in the prevention of migraine. J Int Med Res 1980;8(suppl 3):49-52S.
52 Okasha A, Ghaleb HA, Sadek A. A double blind trial for the clinical management of psychogenic headache. Br J Psychiatry 1973;122:181-3.

53 Pfaffenrath V, Diener HC, Isler H, Meyer C, Scholz E, Taneri Z, et al. Efficacy and tolerability of amitriptylinoxide in the treatment of chronic tension-type headache: a multi-centre controlled study. Cephalalgia 1994;14:149-55.

54 Rampello L, Alvano A, Chiechio S, Malaguarnera M, Raffaele R, Vecchio I, et al. Evaluation of the prophylactic efficacy of amitriptyline and citalopram, alone or in combination, in patients with comorbidity of depression, migraine, and tension-type headache. Neuropsychobiology 2004;50:322-8.

55 Tarasova SV, Amelin AV, Skoromets AA. Fluvoxamine, amitriptyline and transcranial electrostimulation of the brain in the treatment of chronic daily headache. Zhurnal Nevropatoliogii I Psikhiatrii Imeni S S Korsakova 2009;108:43-6.

56 Vernon H, Jansz G, Goldsmith CH, McDermaid C. A randomized, placebo-controlled clinical trial of chiropractic and medical prophylactic treatment of adults with tension-type headache: results from a stopped trial. J Manipulative Physiol Ther 2009;32:344-51.

57 Walker Z, Walker RWH, Robertson MM, Stansfeld S. Antidepressant treatment of chronic tension-type headache: a comparison between fluoxetine and desipramine. Headache 1998;38:523-8.

58 Ziegler DK, Hurwitz A, Hassanein RS, Kodanaz HA, Preskorn SH, Mason J. Migraine prophylaxis. A comparison of propranolol and amitriptyline. Arch Neurol 1987;44:486-9.

59 Okasha A, Ghaleb HA, Sadek A. A double blind trial for the clinical management of psychogenic headache. Br J Psychiatry 1973;122:181-3.

60 Moja PL, Cusi C, Sterzi RR, Canepari C. Selective serotonin re-uptake inhibitors (SSRIs) for preventing migraine and tension-type headaches. Cochrane Database Syst Rev 2005;3:CD002919.

61 Jackson JL, O’Malley PG, Kroenke K. Clinical predictors of mental disorders among medical outpatients. Validation of the "S4" model. Psychosomatics 1988;39:431-6.

62 Kroenke K, Spitzer RL, Williams JB, Linzer M, Hahn SR, deGruy FV, et al. Physical symptoms in primary care. Predictors of psychiatric disorders and functional impairment. Arch Fam Med 1994;3:774-9.

63 Kroenke K, Jackson JL, Chamberlin J. Depressive and anxiety disorders in patients presenting with physical complaints: clinical predictors and outcome. Am J Med 1997;103:339-47.

64 Garvey MJ, Schaffer CB, Tuason VB. Relationship of headaches to depression. Br J Psychiatry 1983;143:544-7.

65 Hendler N. Depression caused by chronic pain. J Clin Psychiatry 1984;45:30-8.

66 Botney M, Fields JL. Amitriptyline potentiates morphine analgesia by a direct action on the central nervous system. Ann Neurol 1983;13:160-4.

67 Eberhard G, Von Knorring L, Nilsson HL, Sundequist U, Wahlander L. Predictors for the outcome of treatment with antidepressants in patients with idiopathic pain syndromes. Nord Psykiatr Tidsskr 1989;43:114-20.

68 Anderson ED. An ascending serotoninergic pain modulation pathway from the dorsal raphe nucleus to the parafascicularis nucleus of the thalamus. Brain Res 1983;269:67-77.

69 Terrin N, Schmid CH, Lau J, Olkin I. Adjusting for publication bias in the presence of heterogeneity. Stat Med 2003;22:2113-26.

Accepted: 9 August 2010 